\title{
Autoeficácia e Desenvolvimento Positivo dos Jovens: Uma Revisão Narrativa da Literatura
}

\author{
Gisele de Rezende Franco*, 1 \\ Orcid.org/0000-0003-3000-6086 \\ Marisa Cosenza Rodrigues ${ }^{1}$ \\ Orcid.org/0000-0001-6711-3198
}

${ }^{1}$ Universidade Federal de Juiz de Fora, Juiz de Fora, MG, Brasil

\begin{abstract}
Resumo
O Desenvolvimento Positivo dos Jovens (PYD) aborda a promoção da saúde de todos os adolescentes, visando potencializar suas capacidades e ressaltar as qualidades positivas desse período evolutivo, combinadas com recursos do ambiente e de pessoas significativas. Dentre tais qualidades, crenças de autoeficácia satisfatórias são essenciais para a obtenção de uma vida saudável, pois configuram-se como recursos promotores de uma adaptação positiva à vida adulta. Objetivou-se na presente revisão narrativa discutir relações entre crenças de autoeficácia e recursos desenvolvimentais em diferentes domínios sociais. Foram recuperados artigos nas bases de dados SciELO, PubMed, Web of Science, Scopus, CAPES e PsycINFO. Empregaram-se os termos de busca autoeficácia e desenvolvimento positivo dos jovens, sem delimitar um intervalo temporal. A produção científica é recente, sendo selecionados 19 estudos que associam autoeficácia e recursos do PYD, com predominância de estudos transversais, e amostras compostas especialmente por jovens do ensino médio. Entre os recursos encontrados, destacam-se competências acadêmicas, físicas, de carreira e suporte social. Isoladamente, autoeficácia e desempenho acadêmico contam com produções científicas mais expressivas, mas a associação entre essas variáveis demanda mais pesquisas. Estudos sobre autoeficácia e PYD são destacados e alguns horizontes são apontados na direção de futuras pesquisas.
\end{abstract}

Palavras-chave: Autoeficácia, desenvolvimento positivo, adolescentes, produção científica.

\section{Self-efficacy and Positive Youth Development: A Narrative Review of the Literature}

\begin{abstract}
Positive Youth Development (PYD) addresses the promotion of the health of all adolescents, aiming to enhance their abilities and highlight the positive qualities of this period of development combined with resources from the environment and from significant people. Among these qualities, satisfactory self-efficacy beliefs are essential for achieving a healthy life, as they constitute resources that promote

* Endereço para correspondência: Rua Doutor Moacir de Castro Xavier, 54, Bairro Linhares, Juiz de Fora, MG, Brasil 36060-490. E-mail: gisele.franco2008@hotmail.com

Apoio Financeiro: bolsista Coordenação de Aperfeiçoamento de Pessoal de Nível Superior (CAPES).
\end{abstract}


a positive adaptation to adult life. This narrative review aimed to discuss relationships between self-efficacy beliefs and developmental resources in different social domains. Articles were retrieved from the SciELO, PubMed, Web of Science, Scopus, CAPES and PsycINFO databases. The search terms 'self-efficacy' and 'positive youth development' were used, without delimiting the time interval. The scientific production was found to be recent, with 19 studies included that associated self-efficacy and PYD resources. These were predominantly cross-sectional studies, with samples mainly composed of high school students. Among the resources found, academic, physical, career and social support skills were highlighted. Individually, self-efficacy and academic performance presented more scientific productions, however, the association between these variables requires more research. Studies on self-efficacy and PYD are highlighted and some horizons for future research are pointed out.

Keywords: Self-efficacy, positive development, adolescents, scientific production.

\section{Autoeficacia y Desarrollo Positivo de los Jóvenes: Una Revisión Narrativa de la Literatura}

\section{Resumen}

El Desarrollo Positivo de los Jóvenes aborda la promoción de la salud de todos los adolescentes, buscando potenciar sus capacidades y cualidades positivas relacionadas con los recursos del ambiente y con las personas significativas. Dentro de estas cualidades, son necesarias las creencias de autoeficacia para lograr una vida saludable, además estas se constituyen en recursos promotores para adaptarse a la vida adulta. En la presente revisión se tuvo como objetivo discutir las relaciones entre autoeficacia y recursos del desarrollo en diferentes dominios sociales. Fueron recuperados artículos en las bases SciELO, PubMed, Web of Science, Scopus, CAPES y PsycINFO. Los términos empleados para la búsqueda fueron autoeficacia y desarrollo positivo de los jóvenes, sin delimitó un intervalo de tiempo. La producción científica es reciente, siendo seleccionados 19 estudios, con predominio de estudios transversales, y muestras compuestas por jóvenes del enseñanza medio. Entre los recursos encontrados se resaltan las competencias académicas, físicas, de carrera e apoyo social. De forma aislada, autoeficacia y desempeño académico cuentan con producciones científicas más expresivas, pero la asociación entre esas dos variables requiere más investigaciones. Estudios sobre autoeficacia y desarrollo positivo se destacan y horizontes se señalan en la dirección de futuras investigaciones.

Palabras clave: Autoeficacia, desarrollo positivo, adolescentes, producción científica.

Investigações relacionadas a autoeficácia em contextos de Desenvolvimento Positivo dos Jovens (PYD do inglês Positive Youth Development) podem subsidiar uma prática profissional qualificada no campo da prevenção, por meio do incremento de qualidades positivas. Bandura (2004) defendeu a compreensão de processos de mudança pessoal relacionados à adoção de práticas favoráveis e à eliminação de hábitos prejudiciais à saúde. Para tanto, são imprescindíveis ações voltadas à promoção da saúde, definida como "o processo de capacitação da comunidade para atuar na melhoria da sua qualidade de vida e saúde, incluindo maior participação no controle deste processo" (World Health Organization [WHO], 1986). Tais ações afirmam um conjunto de valores como a saúde e seus determinantes sociais, a equidade e a participação social, balizando diversas estratégias que visam a constituição de políticas públicas profícuas, como a Política Nacional de Promoção da Saúde, que no Brasil envolve entre suas ações prioritárias: a prevenção e o controle do tabagismo, a prevenção da violência, a redução da morbimortalidade em decorrência do uso abusivo de álcool/drogas, e em função dos acidentes de trânsito (Ministério da Saúde, 2010). 
Especificamente, a autoeficácia, de acordo com Bandura (1977, p. 3), é definida como "a crença que a pessoa tem acerca de sua capacidade para organizar e executar cursos de ação requeridos para alcançar determinados tipos de desempenho". Assim, a crença de autoeficácia representa um mecanismo regulador das ações humanas que impacta diretamente no estabelecimento de metas, na execução de tarefas e na tomada de decisão. Com isso, pessoas com elevada autoeficácia tendem a estabelecer objetivos mais desafiadores, explorar melhor o seu ambiente e persistir na tarefa diante de obstáculos, quando comparadas com pessoas apresentando baixa autoeficácia (Pacico, Ferraz, \& Hutz, 2014; Sbicigo, Teixeira, Dias, \& Dell'Aglio, 2012).

$\mathrm{O}$ estudo desse constructo encontra-se alinhado ao modelo da cognição social, proposto por meio da teoria social cognitiva de Albert Bandura (2008), que prevê o aprendizado, a motivação e o desempenho humanos como atividades complexas, afetadas por variáveis pessoais (conhecimentos, expectativas, atitudes), comportamentais (habilidades, práticas) e socioambientais (normas sociais, influência dos pares), as quais interagem mutuamente. A teoria social cognitiva, assim como a teoria da identidade (Erikson, 1968/1976) e a teoria do bem-estar (Seligman, 2011), para citar outras, culminaram na adoção de modelos mais abrangentes para a compreensão de fenômenos do desenvolvimento. Sob essa perspectiva, despontou a abordagem do Desenvolvimento Positivo dos Jovens (DPJ), iniciada na década de 1990, nos Estados Unidos, sendo definida como o "envolvimento em comportamentos pró-sociais e o evitamento de comportamentos que comprometem e prejudicam a saúde e o futuro" (Lerner \& Galambos, 1998, p. 435).

Conforme preconizado por pesquisadores no âmbito do DPJ (Benson et al., 2006; Catalano, Berglund, Ryan, Lonczak, \& Hawkins, 2004; Damon, 2004; Lerner, Phelps, Forman, \& Bowers, 2009), torna-se imprescindível estudar mais profundamente questões sobre saúde, bem-estar, recursos individuais e do ambiente almejando gerar trajetórias de desenvolvimento mais salutares. Partindo dessa premissa, o DPJ possibilita a criação de programas que podem prevenir comportamentos de risco, e igualmente, promover ambientes acolhedores e sustentadores de atividades adequadas ao desenvolvimento juvenil (Senna \& Dessen, 2012). Damon (2004) destaca que o campo do DPJ abrange os pontos fortes, talentos e competências que a juventude pode cultivar e maneiras de alinhá-los com recursos e suporte de múltiplos domínios sociais (família, escola, grupo de pares, comunidade) para maximizar o desenvolvimento dos indivíduos e da sociedade.

Nessa direção, para alcançar um desenvolvimento promissor os jovens necessitam passar por experiências que priorizem sua participação como protagonistas ou agentes do seu processo de evolução, com vistas a enfrentar as ameaças do ambiente como oportunidades, e escolher estratégias efetivas para lidar com desafios. Para tanto, o incremento da autoeficácia é fundamental na aquisição e manutenção de padrões de conduta adaptativos. Azzi e Polydoro (2006) afirmam que o poder preditivo das crenças de autoeficácia em relação à escolha de tarefas, à motivação ou ao modo como o indivíduo se sente em relação a determinadas atividades evidenciou o conceito de autoeficácia percebida devido ao seu poder explicativo.

Segundo Bandura (1997), a autoeficácia percebida, com a intenção de atingir demandas referentes a adoção de comportamentos relevantes para a saúde e realização de tarefas em diferentes condições, afeta os níveis de estresse e ansiedade do jovem, e, do mesmo modo, os ambientes promotores de saúde (p. e., escolas) contribuem para incrementar o senso de eficácia juvenil. Verifica-se, assim, que tanto as condições do ambiente quanto as ações empregadas para atender às demandas - sejam as condições fisiológicas dos jovens ou as crenças de autoeficácia - interatuam podendo possibilitar (ou não) a resolução da condição estressora e/ou ansiogênica.

É importante destacar que a inclusão da abordagem do desenvolvimento positivo no campo da prevenção ampliou o foco das pesquisas na área e inseriu a promoção de competência em intervenções com ideário positivo (Catalano et al., 2004; Franco \& Rodrigues, 2014; Seligman, 
2011). O estudo sobre o DPJ ao mapear forças e valores positivos dos jovens e do seu entorno social possibilita a melhora dos resultados educacionais e de saúde para a juventude (Benson, 2003). Desse modo, ao incrementar estratégias de promoção de saúde pautadas na promoção da autoeficácia nessa fase torna-se possível potencializar estes resultados, bem como diminuir a vulnerabilidade social dos jovens.

Além disso, existem evidências empíricas demonstrando que o aumento nas competências avaliadas pelos programas focalizando o DPJ acompanha uma diminuição de comportamentos violentos, evasão escolar, uso de álcool e drogas, comportamento sexual de alto risco, bem como dos transtornos de conduta, transtornos alimentares e depressão (Benetti, Ramires, Schneider, Rodrigues, \& Tremarin, 2007; Catalano et al., 2004). Diante da alta incidência desses problemas, a saúde do adolescente tem sido um tema de interesse crescente não apenas entre pesquisadores mas, também, nas agendas das políticas mundiais da Organização Pan-Americana de Saúde (OPAS, 2007) e da WHO (2013).

Por conseguinte, Franco e Rodrigues (2014), Senna e Dessen (2012), também ressaltam a proeminência de temáticas inovadoras com estudos sobre habilidades sociais e habilidades de vida, bem-estar, resiliência, contextos de risco e de proteção, despontando a abertura teórica e técnica para intervenções proativas.

Diante disso, torna-se relevante investir no conhecimento sobre o DPJ, que segundo estudos promovidos por Jacqueline Lerner e cols. (2009) envolve cinco recursos, os "Cinco Cs" a seguir: Competência (visão positiva das próprias ações em domínios específicos - cognitivo, social, acadêmico, físico, carreira, etc.); Confiança (sentimento de valor próprio e de autoeficácia uma noção de que se pode dominar uma situação e produzir resultados positivos); Conexão (vínculos positivos com pessoas e instituições, refletindo trocas bidirecionais entre o indivíduo e a comunidade, escola, família e pares); Caráter (respeito pelas normas sociais e culturais, moralidade e integridade) e, por fim, Cuidado/ Compaixão (sentimento de empatia e simpatia pelos outros).
Ainda nessa perspectiva, Peter Benson (2003; Benson \& Scales, 2009; Benson et al., 2006), diretor do Search Institute em Minneápolis (EUA) indicou 40 recursos desenvolvimentais que os jovens necessitam para atingirem resultados positivos em suas vidas (20 externos e 20 internos). Conforme os achados dos estudos conduzidos, apurou-se que cada um dos 40 recursos promove o desenvolvimento saudável dos adolescentes e seus ambientes. Dentre os 20 recursos "externos" destacam-se: Apoio (família, vizinhança e escola, oferecendo um ambiente amistoso e encorajador); Empoderamento (adultos valorizando os jovens, compreendendo-os como recursos ativos, e dando-lhes papéis úteis na comunidade); Limites e expectativas (definição de regras e consequências claras pela família, escola e comunidade, bem como a existência de uma influência positiva dos pares); Uso construtivo do tempo (engajamento em atividades criativas e participar de programas organizados para jovens).

Dentre os 20 recursos "internos" têm-se: Compromisso com aprender (estar motivado para obter sucesso na escola, e fazer, no mínimo, uma hora de tarefas escolares em casa durante os dias de aula); Valores positivos (ajudar outras pessoas, demonstrar integridade e responsabilidade, bem como valorizar a igualdade); Competências sociais (saber planejar e tomar decisões, possuir competências interpessoais como habilidades de empatia e amizade, e resistir à pressão frente situações de risco); Identidade positiva (ter senso de controle, propósito na vida, otimismo e elevada autoestima). Segundo Santrock (2014), nas pesquisas realizadas no Search Institute, os jovens com mais recursos desenvolvimentais engajavam-se em menos situações de risco (como uso de álcool e tabaco). O autor exemplifica essa questão tomando por base dados de um levantamento contendo mais de 12 mil estudantes do ensino médio, indicando que $53 \%$ dos alunos que apresentavam de 0 a 10 recursos relataram ter usado álcool três ou mais vezes no último mês, ou ter ficado bêbado mais de uma vez nas duas últimas semanas, comparados com apenas $16 \%$ dos alunos com 21 a 30 recursos, ou $4 \%$ dos alunos com 31 a 40 recursos. Pondera-se que 
maior o número de recursos disponíveis, maiores as chances de prevenir comportamentos de risco e promover comportamentos valorizados socialmente como, por exemplo, obter sucesso na escola e cultivar uma boa saúde.

Diante do exposto, este artigo objetiva apresentar uma revisão narrativa das publicações científicas focalizando a relação entre autoeficácia e DPJ, considerando os múltiplos recursos delimitados por Benson (2003) e Lerner et al. (2009), exemplificados anteriormente. Nota-se que iniciativas que exploram tal interface têm surgido como propostas pontuais atreladas a investigações, em sua maioria, dos fatores de risco, e não dos fatores de proteção ao desenvolvimento juvenil (Connor, George, Gullo, Kelly, \& Young, 2011; Hyde, Hankins, Deale, \& Marteau, 2008; Veselska, Geckova, Reijneveld, \& Dijk, 2011). Para tanto, revisões de literatura são pertinentes, pois agregam as informações obtidas por diversas pesquisas, apontam os resultados semelhantes e os contraditórios, as lacunas presentes na literatura e sugestões para pesquisas futuras, permitindo compreender melhor a influência das crenças de autoeficácia sobre o DPJ.

\section{Método}

Para atingir o objetivo deste estudo, realizou-se uma revisão narrativa pretendendo uma síntese da literatura científica vigente. A técnica de revisão narrativa foi utilizada por possibilitar reunir pesquisas com métodos diferentes, obtendo-se como produto final o estado atual do conhecimento disponível em curto espaço de tempo (Grant \& Booth, 2009; Rother, 2007). Para os autores citados essa técnica é adequada para descrever e discutir o desenvolvimento de um dado tema, sob o ponto de vista teórico/ conceitual, fundamentando-se na interpretação e análise crítica da literatura, de forma a favorecer o levantamento de questões e na aquisição e atualização do referido conhecimento.

Foram conduzidas buscas nas bases SciELO, PubMed, Web of Science, Scopus, CAPES e PsycINFO em agosto de 2016. Não foi delimitado um intervalo de tempo para incluir as publicações, uma vez que almejou-se analisar a pro- dução disponível sobre crenças de autoeficácia envolvendo a área do Desenvolvimento Positivo dos Jovens (analisando os variados recursos nela inseridos).

O processo de coleta do material foi executado utilizando-se os termos self-efficacy and positive youth development na língua inglesa, autoeficácia e desenvolvimento positivo dos jovens na língua portuguesa, e autoeficacia y desarrollo positivo de los jóvenes na língua espanhola. $\mathrm{O}$ campo selecionado a se pesquisar foi "assunto" ou "palavra", quando a opção assunto era inexistente. Os resultados de cada um dos termos foram cruzados entre si utilizando-se o operador booleano "and" com a finalidade de restringir a pesquisa aos documentos que apresentavam ambos os termos.

Os critérios para inclusão das publicações nessa revisão foram: pesquisas em que o constructo da autoeficácia fosse o eixo principal. Isto é, estudos contendo essa expressão utilizada nas buscas no título ou palavras-chave, ou ter explícito no resumo que o texto relacionava a autoeficácia com aspectos positivos vinculados ao DPJ. Nessa etapa encontraram-se 125 publicações e, em seguida, foram eliminados documentos que não eram artigos (teses, dissertações, editoriais, livros), artigos repetidos entre as bases de dados, e que abrangiam o público infantil (com amostras de crianças), por não atenderem ao interesse dessa revisão. Como resultado obteve-se 12 artigos, conforme descrito na Tabela 1.

\section{Tabela 1}

Descrição da Busca Bibliográfica nas Bases Virtuais Pesquisadas

\begin{tabular}{ccc}
\hline Banco de dados & Resultados & $\begin{array}{c}\text { Artigos } \\
\text { selecionados }\end{array}$ \\
\hline SciELO & 3 & 1 \\
PubMed & 12 & 0 \\
Web of Science & 10 & 2 \\
SCOPUS & 21 & 2 \\
Capes & 54 & 6 \\
PsycINFO & 25 & 1 \\
\hline Total & 125 & 12 \\
\hline
\end{tabular}


Em um segundo momento, o banco de dados foi sendo complementado com pesquisas dentro do mesmo enfoque, indicadas nas referências bibliográficas dos estudos que permaneceram na revisão, sendo então acrescentadas sete novas publicações após análise de concordância entre as autoras. Por fim, compuseram essa revisão narrativa um total de 19 artigos que atendiam aos critérios de inclusão e exclusão elencados. Como apontado por Grant e Booth (2009), em estudos de revisão narrativa pode-se, ou não, incluir técnicas qualitativas e quantitativas para analisar a produção científica identificada, com isso, optou-se por não utilizá-las no tratamento dos dados apurados nessa revisão. Os artigos selecionados foram lidos na íntegra, analisados criticamente, e registrados em um protocolo elaborado pelas pesquisadoras, que continha um resumo acrescido de comentários. A organização do resumo gerou as seguintes categorias, a saber: autor, ano de publicação, título, local em que a pesquisa foi realizada, tamanho da amostra, domínios de investigação (escola, família, grupo de pares, comunidade), objetivo, delineamento do estudo (longitudinal, seccional), instrumentos de medida para avaliar a autoeficácia e os aspectos intrínsecos e/ou extrínsecos ao DPJ, e, por fim, os principais resultados referentes à relação entre recursos do DPJ e autoeficácia. Com base nessas categorias, as principais informações obtidas foram agrupadas em três eixos temáticos por afinidade metodológica ou teórica, conforme detalhado na seção posterior.

\section{Resultados e Discussão}

A Tabela 2 apresenta os resultados iniciais dos estudos selecionados. Dentre os dezenove estudos alvo dessa revisão, oito foram realizados em contexto nacional. Onze estudos foram conduzidos em contexto internacional, sendo quatro exclusivamente nos EUA e um em concomitância nos EUA e na África, um no México, um na Itália, um na Espanha, um na Malásia, um na Colômbia e um em Israel. No que diz respeito à temporalidade das publicações, constatou-se um período de abrangência correspondente aos anos de 1996 a 2016. Verificou-se que o primei- ro trabalho, indexado pelo Portal CAPES, data de 1996. Contudo, somente a partir de 2009 houve um incremento na produção, com 14 artigos publicados desde então.

\section{Tabela 2}

Quantidade de Estudos Identificados $(N=19)$

$n$

\begin{tabular}{|c|c|}
\hline Nacionalidade & \\
\hline Brasil & 8 \\
\hline EUA & 4 \\
\hline EUA/África & 1 \\
\hline México & 1 \\
\hline Itália & 1 \\
\hline Espanha & 1 \\
\hline Malásia & 1 \\
\hline Colômbia & 1 \\
\hline Israel & 1 \\
\hline Ano & \\
\hline 1996 & 1 \\
\hline 1999 & 1 \\
\hline 2005 & 1 \\
\hline 2006 & 1 \\
\hline 2008 & 1 \\
\hline 2009 & 2 \\
\hline 2011 & 3 \\
\hline 2012 & 1 \\
\hline 2013 & 1 \\
\hline 2014 & 2 \\
\hline 2015 & 3 \\
\hline 2016 & 2 \\
\hline
\end{tabular}

De modo geral, verifica-se que pesquisas associando a autoeficácia aos recursos do DPJ são recentes. Além disso, predominaram estudos internacionais, sinalizando dessa forma a necessidade de maior investigação da temática no contexto brasileiro. As principais informações obtidas, com base nos estudos encontrados, foram inseridas no protocolo mencionado contendo as categorias referidas, agrupadas nos 
três eixos temáticos, a saber: (a) Características metodológicas; (b) Tipo(s) de avaliação da autoeficácia e domínios sociais; e (c) Implicações da associação entre autoeficácia e recursos do desenvolvimento positivo.

\section{Características Metodológicas}

Neste eixo temático são apresentadas as informações quanto ao delineamento dos estudos e características dos participantes. Com relação ao delineamento, a maioria consistiu de pesquisa correlacional de corte seccional, sendo que apenas uma adotou uma metodologia longitudinal (Carvajal et al., 1999). Cabe ressaltar que os estudos correlacionais permitem identificar os fatores preditores da autoeficácia, mas não possibilitam verificar se há uma relação causal entre as variáveis ou a direção dessa relação. Por exemplo, no caso das relações encontradas entre autoeficácia e responsabilidade pessoal e social dos estudantes (Sanmartín, Carbonell, \& Baños, 2011), não se pode afirmar que a responsabilidade pessoal e/ou social dos jovens ocasionou maior frequência de autoeficácia ou, pelo contrário, se elevado senso de autoeficácia os tornava mais responsáveis em qualquer área da vida.

Como apresenta a Tabela 3, o tamanho da amostra variou de 35 (Noronha \& Ambiel, 2008) a 1698 adolescentes (Souza, Rech, Sarabia, Añez, \& Reis, 2013), com média de idade entre 13 e 18 anos. Quanto aos participantes, a maioria dos estudos tinha somente adolescentes como público-alvo, exceto o estudo de Noronha e Ambiel (2008) que compôs sua amostra de adolescentes $(n=35)$ e também por um dos genitores de cada participante $(n=35)$. De modo geral, os estudos foram realizados com jovens de ambos os sexos (meninas e meninos), e o único estudo que envolveu adolescentes e genitores como público-alvo, não coletou os dados demográficos dos genitores, apenas mencionou que houve predominância de mães (67\%).

\section{Tipo(s) de Avaliação da Autoeficácia e Domínios Sociais}

Os estudos variaram quanto ao tipo de crença de autoeficácia investigada, considerando os objetivos propostos em cada um deles, bem como a variabilidade de contextos nos quais essa crença pode ser estudada, por exemplo, no âmbito da saúde e da educação, ou mais geral. Verifica-se a ocorrência de pesquisas em que os jovens se consideram capazes quando avaliam situações específicas, como enfatizado por Carvajal et al. (1999) sobre o adiamento do início da atividade sexual, ou mesmo por Leal et al. (2015) sobre o desenvolvimento de carreira. Contudo, há também estudos focalizando a crença na capacidade de realizar várias atividades independentemente da situação apresentada (Johnson et al., 2012).

Esses achados convergem com os pressupostos de Bandura (1977), ao ressaltar o fato de a autoeficácia estar relacionada a uma determinada situação e, desse modo, direcionar os pesquisadores a construírem medidas de avaliação para momentos específicos, apresentando a autoeficácia como um estado. Entretanto, contrastando com o que propôs inicialmente o próprio Bandura $(1977,2006,2008)$ em seus estudos, a autoeficácia também pode ser generalizada, pois quando orientada a uma circunstância, a mesma pode afetar as crenças na capacidade do sujeito em outra. Nesse sentido, Pacico et al. (2014) definem a autoeficácia geral como a crença, generalizada e estável, na capacidade de lidar com desafios de modo independente da situação que se apresente, sendo a mesma compreendida como uma crença do tipo traço na própria competência.

Conforme mostra a Tabela 3, no tocante aos instrumentos de coleta de dados em geral, predominou o uso de escalas e questionários. Para avaliar a autoeficácia, 17 instrumentos diferentes foram adotados, sendo que os mais utilizados foram: medidas contendo subescalas avaliando a autoeficácia acadêmica: Selfefficacy Scales (Perceived Competence Scale for Children-PCSC; Chung \& Elias, 1996), Escala de Autoeficácia Acadêmica (Guerreiro-Casanova et al., 2011), Cuestionario sobre la Autoeficacia Académica General (Gaxiola \& Armenta, 2016) e Self-efficacy Questionnaire for Children (Yap \& Baharudin, 2016). Em seguida, a Escala de Autoeficácia para Atividades Ocupacionais (EAAOc; Noronha \& Ambiel, 2008; Nunes \& Noronha, 2009, 2011); e a avaliação da 
Tabela 3

Pesquisas que Avaliaram a Autoeficácia e a sua Relação com os Recursos do DPJ

\begin{tabular}{|c|c|c|}
\hline Autores & $\begin{array}{l}\text { Amostra }(n) \\
\text { domínio social }\end{array}$ & $\begin{array}{l}\text { Instrumentos de medida: } \\
\text { 1. Escala de autoeficácia (AE);2. Medidas de recursos do DPJ }\end{array}$ \\
\hline $\begin{array}{l}\text { Braun-Lewensohn } \\
\qquad(2015)\end{array}$ & $n=400 ;{ }^{\mathrm{d}}$ & $\begin{array}{l}\text { 1. Citizens' Efficacy; 2. Sense of Coherence Scale, Questionnaire of } \\
\text { Values, Youth Social Responsibility Scale, Hope Index }\end{array}$ \\
\hline Carvajal et al. (1999) & $n=827 ;{ }^{\mathrm{a}},{ }^{\mathrm{c}}$ & $\begin{array}{l}\text { 1. Questionnaire of Self-efficacy about the Respondents' Confidence } \\
\text { in Refraining from Having Sex; 2. Questionnaire of Delay the Onset } \\
\text { of Sexual Intercourse, Attitudes, Social Norms }\end{array}$ \\
\hline Chung \& Elias (1996) & $n=556 ;{ }^{\text {a }}$ & $\begin{array}{l}\text { 1. Self-efficacy Scales: academic, social and physical; 2. National } \\
\text { Youth Survey of Antisocial and Delinquent Behavior, Life Events } \\
\text { Checklist, Social Competence Scales-Youth }\end{array}$ \\
\hline Contreras et al. (2005) & $n=120 ;{ }^{\text {a }}$ & $\begin{array}{l}\text { 1. Escala AE Generalizada; 2. Escala de Desempenho Acadêmico } \\
\text { Geral e por Áreas }\end{array}$ \\
\hline $\begin{array}{l}\text { Di Fabio \& Kenny } \\
\qquad(2015)\end{array}$ & $n=254 ;{ }^{\text {a }},{ }^{\mathrm{c}}$ & $\begin{array}{l}\text { 1. Career Decision Self-efficacy Scale; 2. Bar-On Emotional Quotient } \\
\text { Inventory, Teacher and Friend Perceived Social Support Scale, Self- } \\
\text { perceived Employability Scale for Students }\end{array}$ \\
\hline $\begin{array}{l}\text { Gaxiola \& Armenta } \\
\qquad(2016)\end{array}$ & $n=250 ;{ }^{\text {a }}$ & $\begin{array}{l}\text { 1. Cuestionario sobre la AE Académica; } 2 \text {. Modelo estrutural de } \\
\text { desempenho acadêmico }\end{array}$ \\
\hline $\begin{array}{l}\text { Gonzalez, Stein, Kiang, } \\
\quad \text { \& Cupito (2014) }\end{array}$ & $n=179 ;{ }^{\text {a }}{ }^{\mathrm{c}}$ & $\begin{array}{l}\text { 1. College-Going Self-efficacy Scale; 2. Psychological Sense of } \\
\text { School Membership Scale, Moods and Feelings Questionnaire, Way } \\
\text { Discrimination Scale, Social Support Scale }\end{array}$ \\
\hline $\begin{array}{l}\text { Guerreiro-Casanova, } \\
\text { Dantas, \& Azzi (2011) }\end{array}$ & $n=534 ;{ }^{\text {a }}, \mathrm{b}$ & $\begin{array}{l}\text { 1. Escala de AE Acadêmica; 2. Inventário de Estratégias de Estudo } \\
\text { e Aprendizagem }\end{array}$ \\
\hline Gushue (2006) & $n=128 ;{ }^{\text {a }}$ & $\begin{array}{l}\text { 1. Career Decision-making Self-efficacy Scale; 2. Ethnic Identity } \\
\text { Measure, Career Outcome Expectations }\end{array}$ \\
\hline Johnson et al. (2012) & $\begin{array}{l}n=473 \text { África, } \\
n=81 \text { EUA; }\end{array}$ & $\begin{array}{l}\text { 1. General Self-efficacy Scale; 2. Multigroup Ethnic Identity } \\
\text { Measure, Diversity Attitudes Scale of the Civic Attitudes and Skills } \\
\text { Questionnaire, Open-ended questions }\end{array}$ \\
\hline $\begin{array}{l}\text { Leal, Melo-Silva, \& } \\
\text { Teixeira (2015) }\end{array}$ & $n=241 ;{ }^{\mathrm{a}}, \mathrm{b}$ & $\begin{array}{l}\text { 1. Inventário de AE em Desenvolvimento da Carreira; 2. Classificação } \\
\text { Econômica Brasil }\end{array}$ \\
\hline Matias et al. (2009) & $n=316 ;{ }^{\text {a }}$ & $\begin{array}{l}\text { 1. Escala de AE Geral; 2. Questionário de Avaliação do Estilo de } \\
\text { Vida, Questionário de Atividades Físicas, Questionário de Mudança } \\
\text { de Comportamento para a Atividade Física }\end{array}$ \\
\hline $\begin{array}{l}\text { Noronha \& Ambiel } \\
\qquad(2008)\end{array}$ & $\begin{array}{c}n=35 \text { jovens, } \\
n=35 \text { genitores; }{ }^{\mathrm{a}}, \mathrm{b}\end{array}$ & $\begin{array}{l}\text { 1. Escala AE para Atividades Ocupacionais; 2. Escala Aconselha- } \\
\text { mento Profissional }\end{array}$ \\
\hline $\begin{array}{l}\text { Nunes \& Noronha } \\
\qquad(2009)\end{array}$ & $n=333 ;{ }^{\mathrm{a}}$ & $\begin{array}{l}\text { 1. Escala AE para Atividades Ocupacionais; 2. Self-directed Search } \\
\text { Career Explorer }\end{array}$ \\
\hline $\begin{array}{l}\text { Nunes \& Noronha } \\
\quad(2011)\end{array}$ & $n=289 ;{ }^{\mathrm{a}}$ & $\begin{array}{l}\text { 1. Escala AE para Atividades Ocupacionais; 2. Self-directed Search } \\
\text { Career Explorer, Escala Aconselhamento Profissional }\end{array}$ \\
\hline Sanmartín et al. (2011) & $n=822 ;{ }^{\text {a }}$ & $\begin{array}{l}\text { 1. Multidimensional Scales of Self-efficacy; 2. Index of Empathy, } \\
\text { Prosocial Behaviour, Contextual Self-responsibility Questionnaire }\end{array}$ \\
\hline Souza et al. (2013) & $n=1698 ;{ }^{\text {a }}$ & $\begin{array}{l}\text { 1. Escala AE para atividade física; 2. Questionário de frequência } \\
\text { semanal de atividade física }\end{array}$ \\
\hline $\begin{array}{l}\text { Ventura \& Noronha } \\
\qquad(2014)\end{array}$ & $n=142 ;{ }^{\mathrm{a}},{ }^{\mathrm{b}}$ & $\begin{array}{l}\text { 1. Escala AE na Escolha Profissional; 2. Inventário Suporte Familiar, } \\
\text { Escalas: Responsividade/Exigência }\end{array}$ \\
\hline $\begin{array}{l}\text { Yap \& Baharudin } \\
\quad(2016)\end{array}$ & $n=802 ;{ }^{\text {a }}$ & $\begin{array}{l}\text { 1. Self-efficacy Questionnaire; 2. Paternal/Maternal Involvement } \\
\text { Scales, Negative Affect Schedule, Students'Life Satisfaction Scale }\end{array}$ \\
\hline
\end{tabular}

${ }^{\mathrm{a}}$ Escolar; ${ }^{\mathrm{b}}$ familiar; ${ }^{\mathrm{c}}$ grupo de pares; ${ }^{\mathrm{d}}$ comunitário. 
autoeficácia para o desenvolvimento de carreira: Career Decision-making Self-efficacy Scale-Short Form (CDMSES-SF; Gushue, 2006), Career Decision Self-efficacy ScaleShort Form (CDSE; Di Fabio \& Kenny, 2015), e Inventário de Autoeficácia em Desenvolvimento da Carreira (CD-SEI; Leal et al., 2015).

Quanto à avaliação dos recursos do desenvolvimento positivo, foram utilizados instrumentos diferentes em cada estudo, como escalas e questionários diversos, sendo que apenas uma pesquisa (Johnson et al., 2012) fez uso de perguntas abertas ou entrevistas com os participantes, almejando avaliar a percepção dos jovens sobre si mesmos (etnia), sua comunidade (atitudes interculturais) e seu cotidiano. Com isso, dentre os principais recursos intrínsecos investigados tem-se: competências acadêmicas, sociais (p. e., empatia), físicas, de carreira; compromisso com aprender; confiança (sentimento de valor próprio, adoção de estilos de vida saudáveis) e valores positivos (responsabilidade). Já entre os recursos extrínsecos, verificou-se: apoio/suporte social (professores, familiares, pares e adultos); respeito pelas normas sociais e culturais; conexão (vínculos positivos com pessoas e instituições, refletidos na participação em protestos sociais) e uso construtivo do tempo (engajamento em atividades criativas).

Com relação ao domínio social de investigação exposto na Tabela 3, 53\% dos estudos envolveu somente o domínio escolar $(n=10)$, como pôde ser observado nas pesquisas realizadas por Chung e Elias (1996) e, Yap e Baharudin (2016), por exemplo. Verificou-se também quatro estudos (Guerreiro-Casanova et al., 2011; Leal et al., 2015; Noronha \& Ambiel, 2008; Ventura \& Noronha, 2014) que destacaram em conjunto os domínios escolar e familiar, bem como três pesquisas (Carvajal et al., 1999; Di Fabio \& Kenny, 2015; Gonzalez et al., 2014) que analisaram o domínio escolar e o grupo de pares, simultaneamente. Por fim, dois estudos enfatizaram o domínio comunitário apenas (Braun-Lewensohn, 2015; Johnson et al., 2012). Diante da multiplicidade de situações (favoráveis ou não) que podem contribuir para o DPJ, faz-se pertinente especialmente a investigação dos aspectos sau- dáveis vivenciados pelos jovens em diferentes domínios sociais. Portanto, estudos envolvendo mais de um domínio social são relevantes, tendo em vista a saúde integral dos adolescentes, bem como sua adaptação à vida adulta.

Ressalta-se que as escolas participantes dos estudos que elegeram o domínio escolar como alvo eram, predominantemente, da rede pública de ensino. Já com relação a análise dos dados, na maioria dos estudos $(n=18)$ encontrados o método utilizado foi quantitativo, com a utilização de análises estatísticas descritivas, análises fatoriais, análises de variância, regressão linear e múltipla, modelos multivariados e estruturais. Entretanto, como citado anteriormente, um estudo (Johnson et al., 2012) recorreu a um método misto, com análise de dados quantitativos e qualitativos através da análise de conteúdo temática e frequencial. Nessa direção, Cerqueira-Silva, Dessen e Costa (2011) rebatem a supremacia do uso de métodos quantitativos, sugerindo a realização de mais estudos que conciliem o uso de métodos mistos, frente à escassez de trabalhos combinando esses métodos, bem como da necessidade de melhor compreensão dos componentes críticos responsáveis pelo DPJ.

\section{Implicações da Associação entre Autoeficácia e Recursos do Desenvolvimento Positivo}

$\mathrm{O}$ conjunto de publicações revelou alguns fatores que, segundo as pesquisas, apresentam relações significativas entre a autoeficácia e o DPJ. Os resultados destas associações são difíceis de serem comparados devido à grande variabilidade de instrumentos utilizados pelos pesquisadores. Essa dificuldade se faz presente, principalmente, no caso da avaliação dos recursos do DPJ, pois segundo Benson et al. (2006) esses recursos podem ser agrupados de acordo com diversos objetivos desenvolvimentais intrínsecos e extrínsecos ao sujeito. Existe uma ampla variedade de competências que podem ser promovidas segundo Catalano et al. (2004), os quais destacam 15 aspectos objetivos (p. e., autoeficácia, crença no futuro, vínculo, competência social). Também Benson et al. (2006) indicam 40 recursos (p. e., apoio, empoderamento, compromisso com 
aprender, identidade positiva), e Lerner e cols. (2009) recomendam cinco recursos (competência, confiança, conexão, caráter, compaixão), o que é refletido nas subescalas dos diferentes instrumentos. Do mesmo modo, no caso da avaliação da autoeficácia foi ressaltado por Bandura (1977), o fato de a autoeficácia estar relacionada a uma determinada situação e, desse modo, direcionar os pesquisadores a construírem medidas de avaliação para momentos específicos, ou competências múltiplas.

Todavia, ao analisar os resultados dos estudos, independentemente do instrumento utilizado, pode-se observar uma aproximação entre os domínios sociais de investigação e as temáticas pertinentes à realidade de cada domínio (escolar, familiar, grupo de pares e comunitário). Constatou-se, em estudos enfatizando competências acadêmicas, um dos recursos do DPJ focalizados no âmbito escolar, que a autoeficácia foi significativamente associada ao desempenho acadêmico em geral, e por áreas, tais como artes, ciências, línguas, social e matemática (Contreras et al., 2005; Gaxiola \& Armenta, 2016). Evidenciou-se ainda, diferenças significativas para as crenças de autoeficácia acadêmica dos estudantes (nas dimensões envolvendo autoeficácia para aprender e atuar na vida escolar) de acordo com o grau de escolaridade dos pais (Guerreiro-Casanova et al., 2011). Portanto, os adolescentes cujos pais tinham nível superior completo apresentaram maior percepção de autoeficácia acadêmica, estando mais motivados a obter sucesso na escola, tendo em vista as referências do seu entorno social. Assim, conforme Benson (2003), tanto recursos intrínsecos (compromisso com aprender) como extrínsecos (suporte social) são desejáveis para o DPJ.

Além disso, no que diz respeito às pesquisas enfocando competências acadêmicas, verificou-se no estudo conduzido por Chung e Elias (1996) que maiores escores de autoeficácia acadêmica, além da participação em diversas atividades pós-escola e eventos de vida positivos (p. e., receber uma medalha de honra, obter aumento salarial) foram preditoras de baixos padrões de problemas comportamentais nos jovens. Esses achados corroboram as evidências encontradas por Catalano et al. (2004), demonstrando que o aumento nas competências avaliadas, no caso de programas focalizando o DPJ, pode corresponder a uma diminuição de comportamentos de risco na adolescência. Igualmente na pesquisa realizada por Yap e Baharudin (2016), constatou-se que a autoeficácia acadêmica correlacionou-se positivamente ao envolvimento parental, ao afeto positivo e a satisfação com a vida, enquanto a autoeficácia social correlacionou-se ao envolvimento parental e ao afeto positivo.

Nesse direção, o direito e o acesso à escola, em princípio, configuram-se como indicadores protetivos para o público juvenil, demonstrando o poder mediador e catalisador das crenças de autoeficácia tanto para o sucesso escolar, quanto para uma percepção positiva do contexto de vida. Logo, é importante ressaltar que as crenças de autoeficácia funcionam como boas preditoras do desempenho para indivíduos que possuem as habilidades básicas exigidas para cumprir determinada tarefa (Bandura, 1997, 2006). Sendo assim, as habilidades são entendidas como um atributo adquirido e mutável, no qual o indivíduo pode exercer controle, por meio da prática e do seu desenvolvimento. Apesar disso, muito pouco tem sido pesquisado especificamente no Brasil a respeito das crenças de autoeficácia acadêmica, e entre outras, principalmente com relação aos jovens estudantes do ensino médio.

No tocante as atividades ocupacionais e interesses profissionais, investigados no âmbito escolar, verificou-se correlações entre autoeficácia e interesses profissionais em função do sexo, com maiores escores entre meninos dos tipos realista e empreendedor, e entre meninas do tipo social (Nunes \& Noronha, 2009). Sugere-se que a confiança na própria capacidade para realizar tarefas ocupacionais esteja vinculada a práticas de socialização diferenciadas pelo sexo, ou seja, provavelmente, professores e outras pessoas estimularam os jovens de forma distinta quanto a essas atividades, e nesse sentido, torna-se necessário que profissionais atuantes no campo da orientação vocacional, por exemplo, reflitam sobre esses achados. Igualmente, Nunes e Noronha (2011) obtiveram correlações significativas entre autoeficácia e tipos de interesses artístico e 
social, sendo que a autoeficácia associada à variável sexo foi preditora dos tipos de interesses realista e social e, das ciências exatas. Já com relação aos interesses profissionais investigados no âmbito familiar e escolar, conforme Noronha e Ambiel (2008), encontrou-se correlação significativa entre autoeficácia e interesse dos pais em ciências exatas. Esses achados sugerem uma tendência à transmissão de pais para filhos de padrões de interesses em ciências exatas. Além disso, no estudo de Ventura e Noronha (2014), a afetividade dos membros da família e a responsividade materna (postura compreensiva da mãe) foi preditora de maior autoeficácia para escolha profissional. Esses achados são apoiados na literatura por Almeida e Melo-Silva (2011), Almeida e Pinho (2008), Benson (2003) ao destacarem que maior a quantidade de suporte familiar, maior poderá ser a percepção da pessoa a respeito da sua capacidade de realizar uma tarefa, destacando-se, assim, a relevância do desenvolvimento de recursos extrínsecos para elevar a autoeficácia.

Especificamente, em relação a um outro recurso intrínseco do DPJ, as competências de carreira, analisadas em relação aos domínios escolar, familiar e o grupo de pares, constatou-se, segundo Leal et al. (2015) uma associação significativa entre a autoeficácia (confiança no planejamento de carreira, na preparação para a procura de emprego e procura efetiva de emprego) e o nível socioeconômico. Os achados revelaram que um ambiente com oportunidades educacionais/culturais mais amplas é desejável, como salientado também por Damon (2004) referindo-se aos recursos de diferentes domínios sociais (escola, família, pares) tendenciados a maximizar o DPJ. Já no estudo de Gushue (2006), a autoeficácia para tomada de decisões na carreira correlacionou-se positivamente com a identidade étnica dos jovens, sugerindo que a identificação dos alunos com seus grupos étnicos, ou seus valores culturais, podem despontar como um aspecto importante no aconselhamento e educação de carreira. Contudo, no estudo de Di Fabio e Kenny (2015) não foi encontrada correlação entre autoeficácia e inteligência emocional, bem como entre autoeficácia e suporte social. Para os pesquisadores, uma possível justificativa seria, que a autoeficácia talvez inclua habilidades específicas na obtenção de informações ocupacionais, planejamento de carreira e tomada de decisão, que vão além das habilidades emocionais e apoio social normalmente ofertado por professores e outros adultos.

No que diz respeito às competências físicas no âmbito escolar, também um recurso intrínseco derivado do DPJ, os resultados encontrados no estudo de Matias et al. (2009), objetivando investigar o estilo de vida, o nível habitual de atividade física e a percepção de autoeficácia dos jovens, indicaram que alta autoeficácia prediz melhor estilo de vida e, que meninos sedentários apresentam maior autoeficácia que meninas sedentárias. Todavia, a prática de atividade física foi relacionada à maior autoeficácia para as meninas. Os autores, ao observarem também que jovens ativos possuem melhor estilo de vida, sugeriram que este comportamento, em conjunto com uma maior percepção de autoeficácia, é importante na formação de hábitos saudáveis de vida. Do mesmo modo, Souza et al. (2013) encontraram associação positiva entre a autoeficácia e a atividade física, porém com mais consistência entre as meninas que entre os meninos. De modo geral, os pesquisadores mencionados inferiram que a elevação dos níveis de autoeficácia na adolescência pode ser um componente importante em estratégias para a promoção da atividade física.

Quanto aos demais estudos encontrados, os mesmos não foram agrupados por temáticas em comum, tendo em vista a diversidade de temas enredados. A partir disso, os resultados e as principais discussões sobre esses estudos são exibidos de modo separado. No estudo de Gonzalez e cols. (2014), houve correlação significativa entre a autoeficácia para continuar os estudos, ou seguir rumo a um curso superior, o senso de conexão com o contexto escolar e o suporte social do grupo de pares. Tais associações positivas sugerem, ainda, que o apoio dos pares parece ter um efeito protetor, por ex., contra a discriminação, pois o estudo envolvia jovens latinos residentes nos EUA. Confirmando esses achados, Bandura (1997) e Benson (2003) afirmam que a influência 
positiva dos pares (um dos recursos extrínsecos do DPJ) é essencial para reconstruir ativamente crenças de autoeficácia na juventude.

Já na pesquisa de Sanmartín et al. (2011), a autoeficácia, o comportamento pró-social e a empatia foram preditoras da responsabilidade pessoal e social dos estudantes, recurso este destacado no DPJ como intrínseco ao sujeito (Benson, 2003). De um ponto de vista prático, o conhecimento das variáveis psicológicas preditoras do DPJ é um primeiro passo, essencial, na elaboração de programas de intervenção visando a promoção da saúde jovem (Benson et al., 2006; Lerner et al., 2009). Portanto, o DPJ começou a influenciar as intervenções que, além de prevenir, passaram a promover o desenvolvimento social, comportamental e cognitivo.

Com relação ao trabalho de Johnson et al. (2012), encontrou-se correlação significativa entre autoeficácia e identidade étnica na amostra geral (África e EUA), e de participantes da Tanzânia e Uganda, bem como entre a variável idade e autoeficácia. Já as questões abertas resultaram em ganhos nas relações/atitudes interculturais e na autoeficácia, segundo relato dos adolescentes. Esses achados reforçam a aplicabilidade dos constructos inter-relacionadas de identidade étnica, autoeficácia e atitudes interculturais, em diferentes contextos. Especificamente, sobre a autoeficácia, são apontados os benefícios de fomentá-la entre os participantes africanos, diante da precariedade de recursos (materiais escolares, atividades extraclasse) nesse contexto, em comparação aos jovens estadunidenses.

Segundo os resultados obtidos por Braun-Lewensohn (2015), um maior senso de autoeficácia para cidadania correlacionou-se com maior engajamento dos jovens, já a percepção de diferentes valores (sociais, culturais) foi preditora de maior autoeficácia para cidadania, engajamento dos jovens e esperança. A partir disso, é relevante enfatizar a importância do uso construtivo do tempo (como engajar-se em atividades criativas), também um recurso extrínseco do DPJ, capaz de viabilizar uma melhorar participação cívica e autoeficácia cidadã entre os adolescentes, como ressaltado por Benson (2003; Benson et al., 2006).
Enfim, no estudo conduzido por Carvajal et al. (1999), o único com delineamento longitudinal, a autoeficácia foi apenas moderadamente preditora do adiamento da primeira relação sexual na adolescência. Os autores sugeriram, que os jovens com atitudes positivas e comprometidos com as normas sociais, e aqueles cujos pais concluíram o ensino superior, eram menos propensos a se envolverem em relações sexuais no período de seguimento (até cerca de 2 anos). Portanto, o caráter - respeito pelas normas sociais/culturais, moralidade e integridade - (Lerner et al., 2009) e, a influência familiar (Benson, 2003), revelaram-se eficazes para o adiamento da primeira relação sexual. Todavia, cabe ainda avaliar esses recursos psicossociais em amostras diferentes quanto ao sexo, etnia e grau de escolarização dos pais, por ex., para que possam indicar quais as estratégias prioritárias a serem assumidas nas intervenções, visando a redução dos comportamentos de risco relacionados à infecção pelo HIV, e a outras doenças sexualmente transmissíveis, bem como a gravidez precoce na adolescência.

Enfim, diante dos resultados aqui reportados revelou-se uma carência de estudos nacionais abordando as relações entre autoeficácia e recursos positivos e promocionais de saúde na adolescência. Evidencia-se, como sinalizado por Coimbra e Fontaine (2015), que os desafios vividos pela atual geração de adolescentes, com períodos de educação familiar e escolar mais prolongados e a transição para a vida adulta também se estendendo, que os jovens necessitam de maior conscientização para lidar com a frustração e os limites impostos pela presença de outras pessoas com direitos e desejos próprios. Compete aos profissionais da saúde e da educação, enquanto agentes promotores de saúde mental e desenvolvimento, aproximarem-se de outros setores sociais para atuarem sobre os determinantes psicossociais inerentes ao processo de desenvolvimento juvenil. Para tanto, cada vez mais é exigido desses profissionais disporem de políticas destinadas ao público jovem, que vem padecendo pela falta de políticas sociais sustentadoras de propostas interventivas proativas nesta importante etapa evolutiva. 


\section{Considerações Finais}

No que concerne às contribuições desse estudo, destaca-se seu caráter inovador, pois existe uma lacuna de estudos sobre a temática abordada, a qual carece de discussão no contexto nacional. Ressalta-se que essa perspectiva mais positiva do desenvolvimento reflete a visão de que, se por um lado, os adolescentes apresentam recursos intrínsecos ou forças, por outro, a escola, a família, os pares e a comunidade fornecem os recursos que podem promover o fortalecimento dos jovens, incluindo as experiências e oportunidades que os contextos podem fornecer para se obter um melhor desempenho no cumprimento das tarefas evolutivas. Nessa perspectiva, as crenças de autoeficácia são consideradas como uma variável chave quando se trata de promover e aprimorar o desempenho em diferentes áreas por possuírem uma relação direta com os processos de aprendizagem.

A presente revisão apresentou estudos com amostras compostas prioritariamente por adolescentes, porém é de extrema relevância um aprofundamento dessa temática com amostras de populações em outras etapas do desenvolvimento. Assim, há que se recomendar que se abranja todo o espectro do curso de vida, preferencialmente por meio de estudos longitudinais, e que não se concentrem as investigações apenas numa dimensão social, como confirmou-se com relação aos estudos selecionados, que focalizaram ora o domínio escolar ora o comunitário, com exclusividade. Cabe ainda destacar, que as pesquisas revisadas utilizaram diferentes instrumentos para a avaliação dos recursos do DPJ e das crenças de autoeficácia, cujos modelos teóricos podem apresentar algumas diferenças.

Para além disso, essa revisão também apresenta limitações, pois existem outros descritores que podem ser utilizados para encontrar mais trabalhos sobre a temática abordada. Portanto, os estudos aqui identificados podem destoar do número de estudos existentes por não se recuperar amplamente as publicações focalizando a relação entre DPJ e autoeficácia. Um outro fator limitante refere-se à dificuldade de se comparar os resultados dos estudos encontrados, tendo em vista a diversidade de instrumentos e/ou recursos detectados, o que não permite realizar generalizações. Contudo, o presente estudo indica que pesquisas investigando a relação entre recursos do DPJ e crenças de autoeficácia de forma mais dialógica podem contribuir para o planejamento, bem como oferecer subsídios para programas proativos juntos aos jovens.

Ainda um fator que contribui para a limitação dos dados aqui apresentados é que as diversas pesquisas elencadas foram realizadas em diferentes países, ou diferentes contextos socioculturais, os quais necessitam ser reinterpretados dentro do contexto brasileiro.

Não obstante, conforme verificado na produção acadêmica pesquisada, esta revisão narrativa demonstrou como as crenças de autoeficácia associam-se a alguns dos recursos positivos do desenvolvimento na adolescência. Os resultados revelaram que a associação entre autoeficácia e recursos do DPJ (p. e., competências acadêmicas, físicas e de carreira; suporte social; respeito por normas sociais e culturais; conexão com pessoas e instituições; valores positivos -responsabilidade; etc.) tem sido alvo de um número restrito de estudos. Isso parece ser decorrente do pouco tempo de inclusão dos conceitos de autoeficácia associados à recursos salutares na literatura científica, visto que dentre as publicações encontradas a primeira correspondeu ao ano de 1996. Portanto, novas investigações relacionadas à temática aqui reportada são de relevância para ampliar as discussões e preencher as lacunas existentes quanto ao incremento de qualidades positivas, e fatores protetores na juventude, de modo a fomentar intervenções positivas, transformar os jovens em pessoas mais proativas para enfrentar adversidades, fazer escolhas saudáveis, e determinar cursos de ação para sua vida.

Conclui-se que a relação entre autoeficácia e os variados recursos do desenvolvimento positivo dos jovens constitui um problema de pesquisa promissor por favorecer a ótica da prevenção e promoção de saúde na adolescência. Como assinalado, o jovem, ao ser constantemente confrontado com situações geradoras de estresse, necessita de uma maior flexibilidade cognitiva, a fim de que, face à realidade em que vive alcan- 
ce uma adaptação mais satisfatória ao enfrentar situações adversas, de modo a superar tais situações dotados de novas competências.

\section{Referências}

Almeida, F. H., \& Melo-Silva, L. L. (2011). Influência dos pais no processo de escolha profissional dos filhos: Uma revisão da literatura. Psico-USF, 16(1), 75-85. Recuperado em http://producao.usp.br/handle/BDPI/6729

Almeida, M. E. G. G., \& Pinho, L. V. (2008). Adolescência, família e escolhas: Implicações na orientação profissional. Psicologia Clínica, 20(2), 173-184. Recuperado em http://www.scielo.br/ $\mathrm{pdf} / \mathrm{pc} / \mathrm{v} 20 \mathrm{n} 2 / \mathrm{a} 13 \mathrm{v} 20 \mathrm{n} 2$

Azzi, R. G., \& Polydoro, S. A. J. (Eds.). (2006). Autoeficácia em diferentes contextos. Campinas, SP: Alínea.

Bandura, A. (1977). Self-efficacy: Toward a unifying theory of behavioral change. Psychological Review, 84, 191-215. doi: http://dx.doi. org/10.1037/0033-295X.84.2.191

Bandura, A. (1997). Self-efficacy: The exercise of control. New York: W. H. Freeman and Company.

Bandura, A. (2004). Health promotion by social cognitive means. Health Education \& Behavior, 31(2), 143-164.

Bandura, A. (2006). Guide for constructing selfefficacy scales. In F. Pajares \& T. C. Urdan (Eds.), Adolescence and education (Vol. 5, pp. 307-337). Greenwich, CT: Information Age.

Bandura, A. (2008). A evolução da teoria social cognitiva. In A. Bandura, R. G. Azzi, \& S. A. J. Polydoro (Eds.), Teoria social cognitiva: Conceitos básicos (pp. 15-41). Porto Alegre, RS: Artmed.

Benetti, S. P. C., Ramires, V. R. R., Schneider, A. C., Rodrigues, A. P. G., \& Tremarin, D. (2007). Adolescência e saúde mental: Revisão de artigos brasileiros publicados em periódicos nacionais. Cadernos de Saúde Pública, 23(6), 1273-1282. doi: 10.1590/S0102-311X2007000600003

Benson, P. L. (2003). Developmental assets and asset building communities: Conceptual and empirical foundations. In R. M. Lerner \& P. L. Benson (Eds.), Developmental assets and asset-building communities: Implications for research, policy, and practice (pp. 19-43). Norwell, MA: Kluwer Academic.

Benson, P. L., \& Scales, P. C. (2009). The definition and preliminary measurement of thriving in adolescence. Journal of Positive Psychology, 4, 85-104. doi: http://dx.doi. org/10.1080/17439760802399240

Benson, P. L., Scales, P. C., Hamilton, S. F., Sesma, A., Jr., Hong, K. L., \& Roehlkepartain, E. C. (2006). Positive youth development so far: Core hypotheses and their implications for policy and practice. Search Institute Insights \& Evidence, 3(1), 1-13.

*Braun-Lewensohn, O. (2015). Sense of coherence, values, youth involvement, civic efficacy and hope: Adolescents during social protest. Social Indicators Research. doi: 10.1007/s11205-0151049-8

*Carvajal, S. C., Parcel, G. S., Banspach, S. W., Basen-Engquist, K., Coyle, K. K., Kirby, D., \& Chan, W. (1999). Psychosocial predictors of delay of first sexual intercourse by adolescents. Health Psychology, 18(5), 443-452. doi: http:// dx.doi.org/10.1037/0278-6133.18.5.443

Catalano, R. F., Berglund, M. L., Ryan, J. A. M., Lonczak, H. S., \& Hawkins, J. D. (2004). Positive youth development in the United States: Research findings on evaluations of positive youth development programs. The Annals of the American Academy of Political and Social Science, 591(1), 98-124. doi: $10.1177 / 0002716203260102$

Cerqueira-Silva, S., Dessen, M. A., \& Costa, A. L. C., Jr. (2011). As contribuições da ciência do desenvolvimento para a psicologia da saúde. Ciência \& Saúde Coletiva, 16(1), 1599-1609. doi: http:// dx.doi.org/10.1590/S1413-81232011000700096

*Chung, H., \& Elias, M. (1996). Patterns of adolescent involvement in problem behaviors: Relationship to self-efficacy, social competence, and life events. American Journal of Community Psychology, 24(6). doi: 10.1007/BF02511034

Coimbra, S., \& Fontaine, A. M. V. G. (2015). Resiliência e habilidades sociais: Reflexões conceituais e práticas para uma nova geração. In Z. A. P. Del Prette, A. B. Soares, C. S. PereiraGuizzo, M. F. Wagner, \& V. B. R. Leme (Eds.), Habilidades sociais: Diálogos e intercâmbios sobre pesquisa e prática (pp. 186-220). Novo Hamburgo, RS: Sinopsys. 
Connor, J. P., George, S. M., Gullo, M. J., Kelly, A. B., \& Young, R. M. (2011). A prospective study of alcohol expectancies and self-efficacy as predictors of young adolescent alcohol misuse. Alcohol and Alcoholism, 46, (2), 161-169. doi: http://dx.doi.org/10.1093/alcalc/agr004

*Contreras, F., Espinosa, J. C., Esguerra, G., Haikal, A., Polanía, A., \& Rodríguez, A. (2005). Autoeficacia, ansiedad y rendimento académico en adolescentes. Perspectivas en Psicología, 1(2), 183-194. Recuperado en http://www.redalyc. org/articulo.oa? $\mathrm{id}=67910207$

Damon, W. (2004). What is positive youth development? The Annals of the American Academy of Political and Social Science, 591(1), 13-24.

*Di Fabio, A., \& Kenny, M. E. (2015). The contributions of emotional intelligence and social support for adaptive career progress among Italian youth. Journal of Career Development, 42(1), 48-59. doi: 10.1177/0894845314533420

Erikson, E. (1976). Identidade, juventude e crise (2. ed.). Rio de Janeiro, RJ: Zahar (Original publicado em 1968)

Franco, G. R., \& Rodrigues, M. C. (2014). Programas de intervenção na adolescência: Considerações sobre o desenvolvimento positivo do jovem. Temas em Psicologia, 22(4), 677-690. doi: 10.9788/TP2014.4-01

*Gaxiola, M. I. B., \& Armenta, M. F. (2016). Factores que influyen en el desarrollo y rendimiento escolar de los jóvenes de Bachillerato. Revista Colombiana de Psicología, 25(1), 63-82. doi: 10.15446/rcp.v25n1.46921

*Gonzalez, L. M., Stein, G. L., Kiang, L., \& Cupito, A. M. (2014). The impact of discrimination and support on developmental competencies in Latino adolescents. Journal of Latina/o Psychology, 2(2), 79-91. doi: 10.1037/lat0000014

Grant, M. J., \& Booth, A. (2009). A typology of reviews: An analysis of 14 review types and associated methodologies. Health Information and Libraries Journal, 26(2), 91-108. doi: 10.1111/j.1471-1842.2009.00848.x

*Guerreiro-Casanova, D. C., Dantas, M. A., \& Azzi, R. G. (2011). Autoeficácia de alunos do ensino médio e nível de escolaridade dos pais. Estudos Interdisciplinares em Psicologia, 2(1), 36-55. doi: http://dx.doi.org/10.5433/22366407.2011v2n1p36
*Gushue, G. V. (2006). The relationship of ethnic identity, career decision-making self-efficacy and outcome expectations among Latino/a high school students. Journal of Vocational Behavior, 68, 85-95. doi: 10.1016/j.jvb.2005.03.002

Hyde, J., Hankins, M., Deale, A., \& Marteau, T. M. (2008). Interventions to increase selfefficacy in the context of addiction behaviours: A systematic literature review. Journal of Health Psychology, 13, (5), 607-623. doi: 10.1177/1359105308090933

*Johnson, L. R., Ha Kim, E., Johnson-Pynn, J. S., Schulenberg, S. E., Balagaye, H., \& Lugumya, D. (2012). Ethnic identity, self-efficacy, and intercultural attitudes in East African and U.S. youth. Journal of Adolescent Research, 27(2), 256-289. doi: 10.1177/0743558411412955

*Leal, M. S., Melo-Silva, L. L., \& Teixeira, M. O. (2015). Crenças para lidar com tarefas de carreira em estudantes do ensino médio. Avaliação Psicológica, 14(1), 125-132. doi: 10.15689/ ap.2015.1401.14

Lerner, R. M., \& Galambos, N. L. (1998). Adolescent development: Challenges and opportunities for research, programs, and policies. Annual Review of Psychology, 49, 413-446.

Lerner, J. V., Phelps, E., Forman, Y., \& Bowers, E. P. (2009). Positive youth development. In R. M. Lerner \& L. Steinberg (Eds.), Handbook of adolescent psychology ( $3^{\text {rd }}$ Ed., pp. 524-558). Hoboken, NJ: Wiley.

*Matias, T. S., Rolim, M. K. S. B., Kretzer, F. L., Schmoelz, C. P., Vasconcellos, D. I. C., \& Andrade, A. (2009). Estilo de vida, nível habitual de atividade física e percepção de autoeficácia de adolescentes. Revista da Educação Físical UEM, 20(2), 235-243. doi: 10.4025/reveducfis. v20i2.6413

Ministério da Saúde. Secretaria de Vigilância em Saúde. Secretaria de Atenção à Saúde. (2010). Política Nacional de Promoção da Saúde (3. ed.). Brasília, DF: Ministério da Saúde.

*Noronha, A. P. P., \& Ambiel, R. A. M. (2008). Fontes de eficácia e interesses profissionais: Relações entre pais e filhos. Evaluar, 8, 3245. Recuperado em https://revistas.unc.edu.ar/ index.php/revaluar/article/view/503/459

*Nunes, M. F. O., \& Noronha, A. P. P. (2009). Auto-eficácia para atividades ocupacionais e interes- 
ses profissionais em estudantes do ensino médio. Psicologia, Ciência e Profissão, 29(1), 102-115. Recuperado em http://www.scielo.br/pdf/pcp/ v29n1/v29n1a09

*Nunes, M. F. O., \& Noronha, A. P. P. (2011). Associações entre auto-eficácia para atividades ocupacionais e interesses em adolescentes. Psicologia: Reflexão e Crítica, 24(1), 1-9. Recuperado em http://www.scielo.br/pdf/prc/ v24n1/v24n1a02

Organização Pan-Americana de Saúde. (2007). Health agenda for the Americas 2008-2017. Panama City, Panama: Author. Retrieved from http://www.paho.org/English/DD/PIN/Health_ Agenda.pdf

Pacico, J. C., Ferraz, S. B., \& Hutz, C. S. (2014). Autoeficácia - Yes we can! In C. S. Hutz (Ed.), Avaliação em psicologia positiva (pp. 111-119). Porto Alegre, RS: Artmed.

Rother, E. T. (2007). Revisão sistemática x revisão narrativa [Editorial]. Acta Paulista de Enfermagem, 20(2), v-vi. doi: http://dx.doi.org/10.1590/ S0103-21002007000200001

*Sanmartín, M. G., Carbonell, A. E., \& Baños, C. P. (2011). Relaciones entre empatía, conducta prosocial, agresividad, autoeficacia $\mathrm{y}$ responsabilidad personal y social de los escolares. Psicothema, 23(1), 13-19. Recuperado en http://www.psicothema.com/PDF/3843.pdf

Santrock, J. W. (2014). Adolescência (14. ed.). Porto Alegre, RS: AMGH.

Sbicigo, J. B., Teixeira, M. A. P., Dias, A. C. G., \& Dell'Aglio, D. D. (2012). Propriedades psicométricas da escala de autoeficácia geral percebida (EAGP). Psico, 43(2), 139-146. Recuperado em https://dialnet.unirioja.es/ servlet/articulo?codigo $=5163218$

Seligman, M. E. P. (2011). Florescer: Uma nova compreensão sobre a natureza da felicidade $e$ do bem-estar. Rio de Janeiro, RJ: Objetiva.

Senna, S. R. C. M., \& Dessen, M. A. (2012). Contribuições das teorias do desenvolvimento humano para a concepção contemporânea da adolescência. Psicologia: Teoria e Pesquisa, 28(1), 101108. Recuperado em http://www.scielo.br/pdf/ $\mathrm{ptp} / \mathrm{v} 28 \mathrm{n} 1 / 13$

*Souza, C. A., Rech, C. R., Sarabia, T. T., Añez, C. R. R., \& Reis, R. S. (2013). Autoeficácia e atividade física em adolescentes de Curitiba, Paraná, Brasil. Cadernos de Saúde Pública, 29(10), 2039-2048. doi: http://dx.doi.org/10.1590/0102$311 \times 00127312$

*Ventura, C. D., \& Noronha, A. P. P. (2014). Autoeficácia para escolha profissional, suporte familiar e estilos parentais em adolescentes. Avaliação Psicológica, 13(3), 317-324. Recuperado em https://dialnet.unirioja.es/ servlet/articulo? codigo $=5115078$

Veselska, Z., Geckova, A. M., Reijneveld, S. A., \& Dijk, J. P. (2011). Self-efficacy, affectivity and smoking behavior in adolescence. European Addiction Research, 17, (4), 172-177. doi: $10.1159 / 000326071$

World Health Organization. (1986, November 21). Ottawa, Canada. In Ottawa Charter for Health Promotion. First International Conference on Health Promotion. Ottawa, Canada: Canadian Public Health Association. Retrieved from http://www.who.int/healthpromotion/conferences/previous/ottawa/en/

World Health Organization. (2013). World health report: Research for universal health coverage. Retrieved from http://www.who.int/whr/en/

*Yap, S. T., \& Baharudin, R. (2016). The relationship between adolescents' perceived parental involvement, self-efficacy beliefs, and subjective well-being: A multiple mediator model. Social Indicators Research, 126, 257-278. doi 10.1007/ s11205-015-0882-0

Recebido: 14/11/2016

$1^{a}$ revisão: $21 / 06 / 2017$ Aceite final: 06/11/2017

(cc)BY (C) O(s) autor(es), 2018. Acesso aberto. Este artigo está distribuído nos termos da Licença Internacional Creative Commons Atribuição 4.0 (http://creativecommons.org/licenses/by/4.0/), que permite o uso, distribuição e reprodução sem restrições em qualquer meio, desde que você dê crédito apropriado ao(s) autor(es) original(ais) e à fonte, fornecer um link para a licença Creative Commons e indicar se as alterações foram feitas. 